\title{
Special Issue: Spheres of Morality
}

\author{
Dagmar Borchers $\cdot$ Svantje Guinebert $\cdot$ Georg Mohr
}

Online publiziert: 23. November 2020

(C) Der/die Autor(en) 2020

\section{Einleitung der Gastherausgeber}

Gemeinhin geht mit jeder der klassischen Moraltheorien (Tugendethik, Konsequentialismus, deontologischer Ethik und Kontraktualismus) der Anspruch einher, eine jeweils umfassende Moralkonzeption zu sein, die für alle einschlägigen moralischen Fragen Antworten parat hält - gemäß dem Prinzip „One size fits it all“. Die einzelnen Moraltheorien werden in ihren diversen Spielarten jeweils mit dem Anspruch weiterentwickelt, für alle Phänomene der Moral (darunter Verpflichtungen, die Idee des Guten, die Relevanz der Tugenden und andere Elemente des moralischen Denkens, Urteilens und Empfindens) eine plausible, aus einem Grundgedanken heraus entwickelte, systematisch-theoretische Einordnung sowie für diverse Anwendungsfelder und -probleme gute und adäquate Entscheidungsverfahren und konkrete Lösungsvorschläge vorlegen zu können. Im Kontext der systematischen Beschreibung des Phänomenbereiches der Moral, der theoretischen Durchdringung und systematischen Ordnung wie auch in Anwendungsfragen reklamiert jede Moraltheorie für sich, die beste, plausibelste und angemessenste zu sein. Das führt bis heute zu einem de facto unentschiedenen Wettstreit der Theorien untereinander, der auch im Bereich der Angewandten Ethik eine wichtige Rolle spielt. Auch hier geht es oftmals nicht nur darum, zu einem moralischen Konflikt systematisch Stellung zu beziehen, sondern explizit oder implizit auch um den Nachweis, dass man nur in dem von der

\footnotetext{
D. Borchers $(\bowtie) \cdot$ S. Guinebert $\cdot$ G. Mohr Institut für Philosophie, Universität Bremen, Enrique-Schmidt-Str. 7, Postfach 330 440, 28359 Bremen, Deutschland

E-Mail: dagmar.borchers@uni-bremen.de

S. Guinebert

E-Mail: svantje.guinebert@uni-bremen.de

G. Mohr

E-Mail: georg.mohr@uni-bremen.de
} 
Autorin oder dem Autor gewählten theoretischen Rahmen zu einer angemessenen Problemdarstellung und Lösung gelangen kann.

Dieser Konkurrenzkampf hat über die Jahrhunderte dazu geführt, dass die klassischen Moraltheorien heute ein breites Spektrum an Varianten entwickelt haben und in Hinblick auf ihre vermeintlichen oder akzeptierten „Schwächen“ mit einer Vielzahl an Ideen zu deren Behebung aufwarten können. Die Diskussion um die Frage, welche Moraltheorie das Phänomen der Moral und des moralischen Denkens am tiefsten durchdrungen hat, kann man vor diesem Hintergrund als einen wichtigen und fruchtbaren „Motor“ der Moralphilosophie und ihrer dynamischen Entwicklung bezeichnen. Der Wettkampf ist aber - und das ist ein relevanter und bedenkenswerter Befund - bis heute unentschieden. Keine Moraltheorie ist aufgrund ihrer Unzulänglichkeiten oder grundsätzlicher Inadäquatheit aufgegeben worden; alle werden weiterhin diskutiert, angewendet und vehement philosophisch verteidigt.

Die Konkurrenzsituation hat dabei durchaus ihre negativen Seiten: Die Frontstellung führt nicht zuletzt zu einer einseitigen Wahrnehmung derjenigen Theorie, mit der man selbst arbeitet. Sie führt dazu, andere Konzeptionen von vornherein aus einer bestimmten Perspektive wahrzunehmen und den Blick dafür zu verlieren, was sie leisten können. Mit dieser theoretisch-systematischen Festlegung ist in Anwendungskontexten immer auch eine vordefinierte Problemwahrnehmung und Beschreibung verbunden, die nur ganz bestimmte Aspekte in den Blick zu nehmen bereit ist. Der Wettbewerb hat zudem in rhetorischer Hinsicht etwas durchaus Ermüdendes.

Darum ist es an der Zeit, einmal die Blickrichtung zu ändern. Wie wäre es, sich von dem Gedanken einer Konkurrenz zu lösen? Warum nicht zugestehen, dass die unterschiedlichen Konzeptionen aus verschiedenen Perspektiven auf das Phänomen des moralischen Denkens und Urteilens blicken und dass jede Perspektive ihre Berechtigung hat? Das hat sie deshalb, weil das Phänomen selbst vielschichtig, vielleicht sogar in sich widersprüchlich ist, sodass jede Moraltheorie bestimmte Aspekte sehr gut erfasst, andere dagegen nicht. Die Blickrichtung zu ändern, würde u. a. bedeuten, eine grundsätzlich wohlmeinende Haltung allen Konzeptionen gegenüber einzunehmen und sich zu fragen, wo jeweils deren Kompetenzbereiche liegen, wie sie einander ergänzen und wie sie in ein konstruktives, einander wechselseitig bereicherndes Verhältnis zueinander gebracht werden könnten. Ähnlich wie Michael Walzer nach Sphären der Gerechtigkeit fragte, in denen unterschiedliche Gerechtigkeitskonzeptionen ihren Ort haben, fragen wir nach Sphären der Moralität, in denen bestimmte Moralkonzeptionen ihre Stärken entfalten könnten.

Die dem Schwerpunkt zugrundliegende Tagung des Instituts für Philosophie an der Universität Bremen im August 2018 war genau dieser Frage gewidmet. Die Intention war, Philosophinnen und Philosophen, die für unterschiedliche Moraltheorien und metaethische Positionen stehen, miteinander ins Gespräch zu bringen, um zu diskutieren, ob das Bild einer wechselseitigen Ergänzung oder gar Synthese der Theorien mit ihren spezifischen Kompetenzen und Kompetenzfeldern möglich und gewinnbringend ist. Sind Tugendethik, Pflichtethik, Konsequentialismus und Kontraktualismus vielleicht nur unterschiedliche Wege, ein und denselben Berg zu erklimmen? Mit dieser Fragestellung greifen Tagung und Schwerpunkt ein Projekt auf, das schon von Henry Sidgwick, Thomas Nagel und Derek Parfit in unterschied- 
licher Weise angedacht worden ist; sie versuchen jedoch, einen Schritt weiter zu kommen.

So knüpft Roger Crisp (Oxford) in seinem Essay zum einen an Sidgwicks Einteilung der metaethischen Grundpositionen in Egoismus, Konsequentialismus und Deontologie und zum anderen an die sokratische Frage „Wie sollen wir leben?“ an, die er für die Hauptfrage der Ethik hält. Angesichts des unversöhnlichen und andauernden Streits zwischen den metaethischen Grundpositionen sieht er die normative philosophische Ethik fatalerweise in einer Paralyse befangen. Fatal, weil die Ethik damit ihren genuinen Sinn verfehle und weil unter den Prämissen der bisherigen Debatten keine konstruktive Überwindung der Situation in Aussicht stehe. Während Crisp sich von Derek Parfits ,triple theory“ nicht ganz überzeugen kann, plädiert er für einen Suspens dogmatischer Ethiktheorien zugunsten einer Einstellung, die er mithilfe einer Analogie illustriert: Einen Weg aus einer dunklen Höhle finden wir nur, wenn wir bereit sind, die Schwächen der eigenen Sichtweise und die Vorteile der Sichtweisen der anderen anzuerkennen.

Das Spektrum der konkurrierenden Metaethiken erweitert Svantje Guinebert (Bremen) gegenüber den von Sidgwick und Crisp genannten Optionen um die Tugendethik und den Kontraktualismus. Egoismus ist ihr zufolge keine alternative Grundposition auf derselben Theorieebene, sondern lediglich eine Spielart des Konsequentialismus. Moraltheorien betrachtet Guinebert hinsichtlich ihrer Merkmale und Funktionen in Analogie zu Sprachen, und sie legt dar, dass die normativen Orientierungen der einzelnen Moraltheorien insofern gut begründet seien, als sie jeweils für verschiedene normative Problemlagen Lösungen ermöglichen. Eben dies rechtfertige auch, dass wir nicht mit einer Theorie alle moralischen Probleme zu lösen versuchen, sondern jeweils die für das betreffende Problem funktional adäquate Moraltheorie in Anschlag bringen sollten. Der Beitrag erläutert, was dies im Einzelnen bedeutet, nicht zuletzt im Hinblick auf das, worum es laut Guinebert vor allem geht: Freiheit.

Wenn es darum geht, das Verhältnis zwischen den grundlegenden metaethischen Theorien neu zu überdenken, erfordert dies auch, die innere Konsistenz der jeweiligen Theorie, ihre Annahmen und Grundbegriffe zu prüfen. Die pointierte These von Christoph Halbig (Zürich) lautet, dass die Tugendethik keinen Anspruch darauf erheben kann, ein distinktes und grundlegendes Kriterium des Richtigen und damit ein drittes Paradigma normativer Ethik auf Augenhöhe mit Konsequentialismus und deontologischen Ansätzen bereitzustellen. Das ergebe sich nicht etwa aus einer argumentativen Überlegenheit der anderen beiden Paradigmen, sondern aus einem Missverhältnis zwischen Tugendtheorie (Tugendbegriff) und Tugendethik. Eine Tugendtheorie, die den Anforderungen einer Tugendethik gerecht würde, führe in Dilemmata und scheitere somit. Umgekehrt sei eine ontologisch und normativ gangbare Tugendtheorie kein geeigneter Ausgangspunkt für eine Tugendethik im Sinne eines eigenständigen Paradigmas normativer Ethik. Das Verhältnis zwischen Tugendbegriff und Tugendethik müsse deshalb neu bestimmt werden.

Rudolf Schüßler (Bayreuth) schlägt vor, den Unterschied zwischen deontologischer und konsequentialistischer Ethik an der jeweils verschiedenen Auffassung von „moralischer Zurechnung“ festzumachen. Der deontologischen Ethik wird bisweilen vorgeworfen, sie impliziere Akteur-relative Werte sowie die moralische „Reinheit““ 
der Akteure. Beides sind aber laut Schüßler keine notwendigen Implikationen. Akteur-Relativität komme in der deontologischen Ethik stattdessen auf der Ebene der Zurechnung ins Spiel. Die moralisch relevanten Werte seien hier universelle Werte, die Zurechnung dagegen sei stets bezogen auf Akteure im Sinne individueller Personen. Demgegenüber unterstelle die konsequentialistische Zurechnungstheorie ein von allen moralisch Handelnden geteiltes moralisches „Konto“.

Die Beiträge zeigen, dass eine fruchtbare metaethische Debatte dann geführt werden kann, wenn man darauf verzichtet, von vermeintlich hinreichend identifizierbaren Prototypen ,der klassischen Moraltheorien“ auszugehen, und stattdessen genau diagnostizierte Annahmen und Behauptungen auf ihre Begründung und Tragweite hin erörtert. Nur so gelangt man zu einer präziseren Einschätzung des jeweils erhobenen moralphilosophischen Anspruchs.

Wir betrachten die Publikation der Beiträge, die alle ein doppelt blindes Begutachtungsverfahren durchlaufen haben, als einen inhaltlichen Auftakt, der bewusst an Impulse, Ideen und Diskussionen anknüpft, die es in dieser Richtung bereits gibt. Wir würden diese Überlegungen gerne fortsetzen und vertiefen und freuen uns, wenn dieses Projekt auch den Leserinnen und Lesern in seiner Intention einleuchtet und ihr Interesse findet.

\section{Introduction by the guest editors}

The extant moral theories - such as consequentialism, virtue ethics, and deontological accounts - are standardly taken to compete with one another; each purports to be the correct global and all-embracing moral conception. In this one-size-fitsall approach, a moral theory is expected to provide answers to any and all moral questions. This position still dominates the ethical discussion. It has been fruitful, since any of these theoretical conceptions has been developed in various aspects in reaction to its critics. At the same time, it has also been problematic, since it may lead to theoretical narrowness and one-sidedness which ignores the plausibility of other ethical approaches. Moreover, the rhetorical attitude of ,downsizing " theories that seem to be misguided has somehow become tiring.

But isn't it much more likely that each of the traditional theories has its own domain of competence? The papers of the Special Issue, originally presented on a conference that took place in 2018 at the Department of Philosophy at the University of Bremen, Germany, are devoted to this question. They examine whether we can bypass the deadlocked controversy as to which is *the* correct moral theory by adopting a picture on which moral theories complement one another and support multifaceted answers to moral questions. According to this alternative approach, our theories might no longer be comprehensive, yet we can perhaps look forward to drawing on their several strengths jointly, in a way that is not possible when we have to choose between them.

While Roger Crisp (Oxford) and Svantje Guinebert (Bremen) are discussing this idea by looking at different moral theories, Christoph Halbig (Zürich) is concerned with modern virtue ethics and the concept of virtue. Finally, Rudolf Schüßler 
(Bayreuth) compares deontology and consequentialism with regard to agent-relative value, accountability and accounting.

We, the guest-editors and organizers of the conference, hope that the readers will find interest in our project in which we reflect upon and include steps that already have been taken throughout the history of moral philosophy. We also hope that they will enjoy reading the contributions of this Special Issue.

Funding Open Access funding enabled and organized by Projekt DEAL.

Open Access Dieser Artikel wird unter der Creative Commons Namensnennung 4.0 International Lizenz veröffentlicht, welche die Nutzung, Vervielfältigung, Bearbeitung, Verbreitung und Wiedergabe in jeglichem Medium und Format erlaubt, sofern Sie den/die ursprünglichen Autor(en) und die Quelle ordnungsgemäß nennen, einen Link zur Creative Commons Lizenz beifügen und angeben, ob Änderungen vorgenommen wurden.

Die in diesem Artikel enthaltenen Bilder und sonstiges Drittmaterial unterliegen ebenfalls der genannten Creative Commons Lizenz, sofern sich aus der Abbildungslegende nichts anderes ergibt. Sofern das betreffende Material nicht unter der genannten Creative Commons Lizenz steht und die betreffende Handlung nicht nach gesetzlichen Vorschriften erlaubt ist, ist für die oben aufgeführten Weiterverwendungen des Materials die Einwilligung des jeweiligen Rechteinhabers einzuholen.

Weitere Details zur Lizenz entnehmen Sie bitte der Lizenzinformation auf http://creativecommons.org/ licenses/by/4.0/deed.de. 